Journal of Applied AnALysis

Vol. 11, No. 2 (2005), pp. 171-186

\title{
MAXIMAL SOLUTIONS AND EXISTENCE THEORY FOR FUZZY DIFFERENTIAL AND INTEGRAL EQUATIONS
}

\author{
R. P. AGARWAL, D. O'REGAN and V. LAKSHMIKANTHAM \\ Received May 27, 2003 and, in revised form, October 4, 2004
}

\begin{abstract}
New existence results are presented for fuzzy differential and integral equations. Our analysis combines the stacking theorem with results concerning the maximal solution for an appropriate differential equation.
\end{abstract}

\section{Introduction}

We consider fuzzy sets with respect to a nonempty base set $X$ (usually $\left.X=\mathbb{R}^{n}\right)$. To each $x \in X$ we assign a membership grade $u(x)$ taking values in $[0,1]$ with $u(x)=0$ corresponding to non-membership, $0<u(x)<1$ to partial membership and $u(x)=1$ to full membership. For example a fuzzy set $u \in E^{n}$ is a function $u: \mathbb{R}^{n} \rightarrow[0,1]$ for which

(i) $u$ is normal;

(ii) $u$ is fuzzy convex;

(iii) $u$ is upper semicontinuous;

2000 Mathematics Subject Classification. 47S40.

Key words and phrases. Existence results, fuzzy differential and integral equations, maximal solution, stacking theorem.

ISSN 1425-6908 C Heldermann Verlag. 
and

(iv) the closure of $\left\{x \in \mathbb{R}^{n}: u(x)>0\right\}$, denoted $[u]^{0}$ and called the support, is compact.

For $0<\alpha \leq 1$ the $\alpha$-level set $[u]^{\alpha}$ is defined by

$$
[u]^{\alpha}=\left\{x \in \mathbb{R}^{n}: u(x) \geq \alpha\right\}
$$

and note the support is $\overline{\bigcup_{\alpha \in(0,1]}[u]^{\alpha}}$. Clearly $[u]^{\alpha} \in C K\left(\mathbb{R}^{n}\right)$ for $\alpha \in[0,1]$ (here $C K\left(\mathbb{R}^{n}\right)$ denote the family of nonempty, convex, compact subsets of $\left.\mathbb{R}^{n}\right)$. We define $D: E^{n} \times E^{n} \rightarrow[0, \infty)$ by

$$
D(u, v)=\sup \left\{d_{H}\left([u]^{\alpha},[v]^{\alpha}\right): \alpha \in[0,1]\right\} ;
$$

here $d_{H}$ is the Hausdorff distance (i.e. the Hausdorff distance between two nonempty bounded subsets $A$ and $B$ of a metric space $(X, d)$ is

$$
d_{H}(A, B)=\max \left\{\sup _{a \in A} d(a, B), \sup _{b \in B} d(b, A)\right\}
$$

where $\left.d(a, B)=\inf _{b \in B} d(a, b)\right)$. We let $\hat{0}$ be the fuzzy set defined by $\hat{0}(x)=1$ if $x=0$ and $\hat{0}(x)=0$ if $x \neq 0$.

Fix $T>0$ and let $C_{n}[0, T]$ be the space of continuous functions $f:[0, T] \rightarrow \mathbb{R}^{n}$ with the usual norm i.e. $|f|_{0}=\sup _{t \in[0, T]}|f(t)|$ for $f \in C_{n}[0, T]$. Denote the space of normal connected (i.e. the $\alpha$-level sets are connected) upper semicontinuous fuzzy sets with compact support over $C_{n}[0, T]$ by $C^{n}[0, T]$ and we give it the metric $D_{0}$ induced by the Hausdorff metric on compact subsets of $C_{n}[0, T]$ (i.e. $D_{0}(u, v)=$ $\sup \left\{d_{H}\left([u]^{\alpha},[v]^{\alpha}\right): \alpha \in[0,1]\right\}$ where $d(z, w)=|z-w|_{0}$ for $z \in[u]^{\alpha}$ and $w \in[v]^{\alpha}$ with $\left.u, v \in C^{n}[0, T]\right)$. The compact sets here are characterized by the Arzela-Ascoli theorem, as families of continuous functions on $[0, T]$ which are equicontinuous and uniformly bounded. By $L_{n}^{1}[0, T]$ we mean the space of integrably functions from $[0, T]$ to $\mathbb{R}^{n}$ with the usual norm i.e. $|f|_{1}=\int_{0}^{T}|f(t)| d t$ for $f \in L_{n}^{1}[0, T]$. By $L_{n}^{\infty}[0, T]$ we mean the space of measurable functions from $[0, T]$ to $\mathbb{R}^{n}$ bounded almost everywhere on $[0, T]$

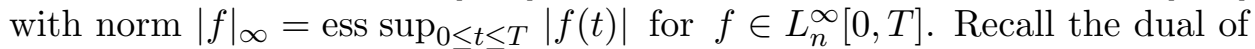
$L_{n}^{1}[0, T]$ (i.e $\left.\left(L_{n}^{1}[0, T]\right)^{\prime}\right)$ is $L_{n}^{\infty}[0, T]$ and $C_{n}[0, T]$ is a closed subspace of $L_{n}^{\infty}[0, T]$. A sequence $\left\{x_{n}\right\}$ in a Banach space $X$ is said to converge weakly to $x \in X$ if $f\left(x_{n}\right) \rightarrow f(x)$ for all $f \in X^{\prime}$. A sequence $\left\{f_{n}\right\}$ in $X^{\prime}$ converges weak $^{\star}$ to $f \in X^{\prime}$ if $f_{n}(x) \rightarrow f(x)$ for all $x \in X$. If we supply $L_{n}^{\infty}[0, T]$ with the weak ${ }^{\star}$ topology then we let $A_{n}[0, T]=\left\{f \in C_{n}[0, T]: f^{\prime} \in L_{n}^{\infty}[0, T]\right\}$. If we supply $L_{n}^{p}[0, T]$ (here $1 \leq p<\infty$ ) with the weak topology then we let $A_{n}^{p}[0, T]=\left\{f \in C_{n}[0, T]: f^{\prime} \in L_{n}^{p}[0, T]\right\}$.

In Section 2 we look at the structure of solution sets for differential and integral inclusions. The results in this section are new and extend previously known results in the literature (see $[1,6,9]$ and the references therein). Next 
we recall the results from the literature $[2,3]$ which will be used in Section 2. First we consider the differential inclusion

$$
\left\{\begin{array}{l}
y^{\prime}(t) \in F(t, y(t)) \text { for a.e. } t \in[0, T] \\
y(0) \in M \subseteq \mathbb{R}^{n}
\end{array}\right.
$$

here $F:[0, T] \times \mathbb{R}^{n} \rightarrow C K\left(\mathbb{R}^{n}\right)$ and $M \subseteq \mathbb{R}^{n}$ is nonempty and compact (respectively nonempty compact and connected). Let $S_{D}\left(M ; \mathbb{R}^{n}\right)$ denote the solution set of (1.1). For our results we will assume the following conditions are satisfied:

$$
\begin{gathered}
x \mapsto F(t, x) \text { is upper semicontinuous for a.e. } t \in[0, T] \\
t \mapsto F(t, x) \text { is measurable for every } x \in \mathbb{R}^{n} \\
\left\{\begin{array}{l}
\text { for each } r>0 \text { there exists } g_{r} \in L^{1}[0, T] \text { with } \\
|F(t, x)| \leq g_{r}(t) \text { for a.e. } t \in[0, T] \text { and every } \\
x \in \mathbb{R}^{n} \text { with }|x| \leq r
\end{array}\right.
\end{gathered}
$$

and

$$
\left\{\begin{array}{l}
\exists M_{0}>\sup \left\{\left|y_{0}\right|: y_{0} \in M\right\} \text { with }|y|_{0}=\sup _{t \in[0, T]}|y(t)|<M_{0} \\
\text { for any possible solution to (1.1). }
\end{array}\right.
$$

Let $\varepsilon>0$ be given and let $\tau_{\varepsilon}: \mathbb{R}^{n} \rightarrow[0,1]$ be the Urysohn function for

$$
\left(\bar{B}\left(0, M_{0}\right), \quad \mathbb{R}^{n} \backslash B\left(0, M_{0}+\varepsilon\right)\right)
$$

such that $\tau_{\varepsilon}(x)=1$ if $|x| \leq M_{0}$ and $\tau_{\varepsilon}(x)=0$ if $|x| \geq M_{0}+\varepsilon$. Let $\tilde{F}(t, x)=\tau_{\varepsilon}(x) F(t, x)$ and consider the problem

$$
\left\{\begin{array}{l}
y^{\prime}(t) \in \tilde{F}(t, y(t)) \text { for a.e. } t \in[0, T] \\
y(0) \in M \subseteq \mathbb{R}^{n} .
\end{array}\right.
$$

Theorem 1.1 ([3]). Suppose $M \subseteq \mathbb{R}^{n}$ is nonempty and compact (respectively nonempty compact and connected) and assume (1.2), (1.3), (1.4) and (1.5) hold. Let $\varepsilon>0$ be given and suppose

$$
|w|_{0}<M_{0} \text { for any possible solution } w \text { to (1.6). }
$$

Then $S_{D}\left(M ; \mathbb{R}^{n}\right)$ is a nonempty compact (respectively nonempty compact and connected) subset of $A_{n}^{1}[0, T]$.

Remark 1.1. Suppose (1.4) is replaced by

$$
\left\{\begin{array}{l}
\text { for each } r>0 \text { there exists } M_{r}>0 \text { with } \\
|F(t, x)| \leq M_{r} \text { for a.e. } t \in[0, T] \text { and every } \\
x \in \mathbb{R}^{n} \text { with }|x| \leq r
\end{array}\right.
$$


and assume (1.2), (1.3), (1.5) and (1.7) hold. Then $S_{D}\left(M ; \mathbb{R}^{n}\right)$ is a nonempty compact (respectively nonempty compact and connected) subset of $A_{n}[0, T]$ (see $\left.[3]\right)$.

Next we consider the integral inclusion

$$
y(t) \in h(t)+\int_{0}^{t} k(t, s) F(s, y(s)) d s \quad \text { for } t \in[0, T]
$$

for any $h \in M \subseteq C_{n}[0, T]$; here $M$ is a nonempty compact (respectively nonempty compact and connected) subset of $C_{n}[0, T], F:[0, T] \times \mathbb{R}^{n} \rightarrow$ $C K\left(\mathbb{R}^{n}\right)$ and the matrix valued function $k:\{(s, t): 0 \leq s \leq t \leq T\} \rightarrow$ $L_{n \times n}^{1}[0, T]$. Let $S_{I}\left(M ; \mathbb{R}^{n}\right)$ denote the solution set of (1.9). For our result we will assume (1.2), (1.3) and (1.4) hold and in addition we suppose the following conditions are satisfied:

$$
\begin{gathered}
\left\{\begin{array}{l}
\text { for each } t \in[0, T], k(t, s) \text { is measurable on }[0, t] \text { and } \\
k(t)=\operatorname{ess} \sup |k(t, s)|, 0 \leq s \leq t, \text { is bounded on }[0, T]
\end{array}\right. \\
\left\{\begin{array}{l}
\text { the map } t \mapsto k_{t} \text { is continuous from }[0, T] \text { to } \\
L^{\infty}\left([0, T], L_{n \times n}^{1}[0, T]\right) ; \text { here } k_{t}(s)=k(t, s)
\end{array}\right.
\end{gathered}
$$

and

$$
\left\{\begin{array}{l}
\exists M_{0}>\sup \left\{|h|_{0}: h \in M\right\} \text { with }|y|_{0}<M_{0} \\
\text { for any possible solution to (1.9). }
\end{array}\right.
$$

Let $\varepsilon>0$ be given and let $\tau_{\varepsilon}: \mathbb{R}^{n} \rightarrow[0,1]$ be as before. Let $\tilde{F}(t, x)=$ $\tau_{\varepsilon}(x) F(t, x)$ and consider the problem

$$
y(t) \in h(t)+\int_{0}^{t} k(t, s) \tilde{F}(s, y(s)) d s \quad \text { for } t \in[0, T]
$$

for any $h \in M$.

Theorem 1.2 ([2]). Suppose $M \subseteq C_{n}[0, T]$ is nonempty and compact (respectively nonempty compact and connected) and assume (1.2), (1.3), (1.4), (1.10), (1.11) and (1.12) hold. Let $\varepsilon>0$ be given and suppose

$$
\left\{\begin{array}{l}
|w|_{0}<M_{0} \text { for any possible solution } w \\
\text { to }(1.13)(\text { for any } h \in M) .
\end{array}\right.
$$

Then $S_{I}\left(M ; \mathbb{R}^{n}\right)$ is a nonempty compact (respectively nonempty compact and connected) subset of $C_{n}[0, T]$. 
In Section 2 we show that $S_{D}\left(M ; \mathbb{R}^{n}\right)$ and $S_{I}\left(M ; \mathbb{R}^{n}\right)$ is a continuum (in the appropriate space) if our nonlinearity $F$ is bounded by a $L^{1}$-Carathéodory function $g$ and if the ordinary differential equation

$$
\left\{\begin{array}{l}
v^{\prime}(t)=a g(t, v(t)) \text { for a.e. } t \in[0, T] \\
v(0)=a_{0}
\end{array}\right.
$$

has a maximal solution (here $a=1$ and $a_{0}=\sup \left\{\left|y_{0}\right|: y_{0} \in M\right\}$ for (1.1) whereas $a=\sup _{t \in[0, T]} k(t)$ and $a_{0}=\sup \left\{|h|_{0}: h \in M\right\}$ for (1.9)). Recall a function $g:[0, T] \times \mathbb{R} \rightarrow \mathbb{R}$ is a $L^{1}$-Carathéodory function if

(a) the map $t \mapsto g(t, y)$ is measurable for all $y \in \mathbb{R}$;

(b) the map $y \mapsto g(t, y)$ is continuous for a.e. $t \in[0, T]$;

and

(c) for any $r>0$ there exists $\mu_{r} \in L^{1}[0, T]$ such that $|y| \leq r$ implies $|g(t, y)| \leq \mu_{r}(t)$ for a.e. $t \in[0, T]$.

In Section 3 we discuss fuzzy differential and integral equations. First we discuss fuzzy Volterra integral equation. Let $V \in C^{n}[0, T]$ (i.e. $V$ is a connected (i.e. the $\alpha$-level sets are connected) upper semicontinuous fuzzy set with compact support over $C_{n}[0, T]$, so the $\beta$-level sets are equibounded and equicontinuous connected sets of continuous functions). Suppose that $V(t)$ is the value at $t$ and $F:[0, T] \times \mathbb{R}^{n} \rightarrow E^{n}$. We consider the fuzzy Volterra integral equation

$$
x(t)=V(t)+\int_{0}^{t} k(t, s) F(s, x(s)) d s
$$

where we interpret $[7,10,13]$ the equation (1.15) as a family of integral inclusions

$$
x_{\beta}(\cdot) \in V_{\beta}+\int_{0}^{\cdot} k(\cdot, s) F_{\beta}\left(s, x_{\beta}(s)\right) d s, \quad 0 \leq \beta \leq 1,
$$

where the subscript $\beta$ indicates that the $\beta$-level set of a fuzzy set is involved (the system (1.16) can only have any significance as a replacement for (1.15) if the solutions generate fuzzy sets). Note (1.16) (for fixed $\beta$ ) will be understood to mean that there exists $h \in V_{\beta}$ such that $x_{\beta}(t) \in h(t)+\int_{0}^{t} k(t, s) F_{\beta}\left(s, x_{\beta}(s)\right) d s$. In [7], (1.15) and (1.16) was discussed if $F$ satisfies the following "global" boundedness assumption:

$$
\left\{\begin{array}{l}
\text { there exists } p \in L^{1}[0, T] \text { with } D(F(t, x), \hat{0}) \leq|p(t)| \\
\text { for a.e. } t \in[0, T] \text { and } x \in \mathbb{R}^{n} ; \text { here } \hat{0} \text { is the fuzzy } \\
\text { set defined by } \hat{0}(x)=1 \text { if } x=0 \text { and } \hat{0}(x)=0 \text { if } x \neq 0 .
\end{array}\right.
$$

This condition is very restrictive from an application viewpoint since $F$ must be integrably bounded for all $x \in \mathbb{R}^{n}$. In Section 3 we remove this 
condition and replace it with a "local" integrably boundedness assumption

$$
\left\{\begin{array}{l}
\text { there exists a } L^{1} \text {-Carathéodory function } \\
g:[0, T] \times[0, \infty) \rightarrow[0, \infty) \text { such that } D(F(t, x), \hat{0}) \leq g(t,|x|) \\
\text { for a.e. } t \in[0, T] \text { and all } x \in \mathbb{R}^{n},
\end{array}\right.
$$

which is exactly what one needs from an application viewpoint. To achieve this we will use the new results for solution sets established in Section 2.

Also in Section 3 we discuss the fuzzy differential equation

$$
\left\{\begin{array}{l}
x^{\prime}(t)=F(t, x(t)) \text { for a.e. } t \in[0, T] \\
x(0)=X_{0} \in E^{n}
\end{array}\right.
$$

where $F:[0, T] \times \mathbb{R}^{n} \rightarrow E^{n}$. We interpret $[8,10,13]$ the equation (1.17) as a family of differential inclusions

$$
\left\{\begin{array}{l}
x_{\beta}^{\prime}(t) \in F_{\beta}\left(t, x_{\beta}(t)\right) \equiv\left[F\left(t, x_{\beta}(t)\right)\right]^{\beta} \\
x_{\beta}(0)=x_{0, \beta} \in\left[X_{0}\right]^{\beta}
\end{array}\right.
$$

where the subscript $\beta$ indicates that the $\beta$-level set of a fuzzy set is involved (the system (1.18) can only have any significance as a replacement for (1.17) if the solutions generate fuzzy sets).

Finally in this section we recall the following results which will be needed in the next section. The first result can be found in [6, Section 4.7], the second in $[13,14]$, the third in $[8,13]$, the fourth in [5], and the fifth in [11].

Theorem 1.3. Define the linear operator $W$ by

$$
(W g)(t)=\int_{0}^{t} k(t, s) g(s) d s \quad\left(\text { here } g \in L_{n}^{1}[0, T]\right)
$$

with (1.10) and (1.11) holding. If $\left\{g_{m}\right\} \subseteq L_{n}^{1}[0, T]$ with $g_{m} \rightarrow g$ weakly in $L_{n}^{1}[0, T]$ as $m \rightarrow \infty$, then there exists a subsequence $S$ of $\{1,2, \ldots\}$ with $W g_{m} \rightarrow W g$ in $C_{n}[0, T]$ as $m \rightarrow \infty$ in $S$.

Theorem 1.4 (Stacking Theorem). Let $\left\{Y_{\beta} \subseteq \mathbb{R}^{n}: 0 \leq \beta \leq 1\right\}$ be a family of subsets satisfying:

(i) $Y_{\beta} \in K\left(\mathbb{R}^{n}\right)$ for all $0 \leq \beta \leq 1$ (here $K\left(\mathbb{R}^{n}\right)$ denotes the family of nonempty compact connected (respectively nonempty compact) subsets of $\mathbb{R}^{n}$ );

(ii) $Y_{\beta} \subseteq Y_{\alpha}$ for $0 \leq \alpha \leq \beta \leq 1$;

and

(iii) $Y_{\beta}=\bigcap_{i=1}^{\infty} Y_{\beta_{i}}$ for any nondecreasing sequence $\beta_{i} \rightarrow \beta$ in $[0,1]$. 
Then there exists a fuzzy set $u \in D^{n}$ such that $[u]^{\beta}=Y_{\beta}$; here $D^{n}$ denotes the set of upper semicontinuous normal connected (i.e. the $\beta$-level sets are connected) fuzzy sets on $\mathbb{R}^{n}$ with compact support (respectively $D^{n}$ denotes the set of upper semicontinuous normal fuzzy sets on $\mathbb{R}^{n}$ with compact support).

Remark 1.2. The conclusion of the stacking theorem is valid if the $Y_{\beta}$ 's are subsets of $C_{n}[0, T]$ and then $u \in C^{n}[0, T]$ if $K(X)$ denotes the family of nonempty compact connected subsets of $X$. Also the conclusion is valid if the $Y_{\beta}$ 's are subsets of $A_{n}[0, T]$ or $A_{n}^{1}[0, T]$.

Theorem 1.5. Let $\Omega$ be an open subset of $\mathbb{R} \times \mathbb{R}^{n}$ and suppose $G$ is an upper semicontinuous map from $\Omega$ to $E^{n}$. Define $F(\cdot, \cdot, \beta): \Omega \rightarrow$ $C K\left(\mathbb{R}^{n}\right)$ to be the mapping $(t, x) \mapsto[G(t, x)]^{\beta}$ (here $\left.0 \leq \beta \leq 1\right)$. Then $F(\cdot, \cdot, \beta)$ is upper semicontinuous on $\Omega$.

Theorem 1.6 (Banach-Alaoglu). The unit ball in the dual of a normed space is compact in the weak topology. Thus the unit ball in $L_{n}^{\infty}[0, T]=$ $\left(L_{n}^{1}[0, T]\right)^{\prime}$ is weak $k^{\star}$ compact.

Theorem 1.7. Let $(X, d)$ be a metric space and $\left\{S_{m}: m \in\{1,2, \ldots\}\right\}$ a sequence of nonempty compact (connected) sets with $S_{m+1} \subseteq S_{m}$ for $m \in\{1,2, \ldots\}$. Then $\bigcap_{m=1}^{\infty} S_{m}$ is a nonempty compact (connected) set.

\section{Solution sets for differential and integral inclusions}

In this section we use Theorem 1.1 (respectively Theorem 1.2) and put natural assumptions (from an application viewpoint) on $F$ to guarantee that $S_{D}\left(M ; \mathbb{R}^{n}\right)$ (respectively $S_{I}\left(M ; \mathbb{R}^{n}\right)$ ) is a continuum.

Theorem 2.1. Suppose $M \subseteq \mathbb{R}^{n}$ is nonempty and compact (respectively nonempty compact and connected) and assume (1.2) and (1.3) hold. In addition suppose the following conditions are satisfied:

$$
\left\{\begin{array}{l}
\text { there exists a } L^{1} \text {-Carathéodory function } \\
g:[0, T] \times[0, \infty) \rightarrow[0, \infty) \text { such that }|F(t, x)| \leq g(t,|x|) \\
\text { for a.e. } t \in[0, T] \text { and all } x \in \mathbb{R}^{n}
\end{array}\right.
$$

and

$$
\begin{aligned}
& \left\{\begin{array}{l}
\text { the problem } \\
\left\{\begin{array}{l}
v^{\prime}(t)=g(t, v(t)) \text { for a.e. } t \in[0, T] \\
v(0)=\max \left\{\left|w_{0}\right|: w_{0} \in M\right\}
\end{array}\right. \\
\text { has a maximal solution } r(t) \text { on }[0, T] .
\end{array}\right.
\end{aligned}
$$


Then $S_{D}\left(M ; \mathbb{R}^{n}\right)$ is a nonempty compact (respectively nonempty compact and connected) subset of $A_{n}^{1}[0, T]$.

Proof. Let $\varepsilon>0$ be given and $M_{0}=\sup _{t \in[0, T]} r(t)+1=r(T)+1$. We will show any possible solution $u$ of (1.1) satisfies $|u|_{0}<M_{0}$ and any possible solution $y$ of (1.6) satisfies $|y|_{0}<M_{0}$. If this is true then Theorem 1.1 guarantees the result. Suppose $u$ is a possible solution of (1.1) and assume $u(0)=x_{0} \in M$. Let $t \in[0, T]$ and we will show $|u(t)|<M_{0}$. For convenience we let $a_{0}=\max \left\{\left|w_{0}\right|: w_{0} \in M\right\}$. If $|u(t)| \leq a_{0}$ we are finished so it remains to discuss the case when $|u(t)|>a_{0}$. In this case since $|u(0)|=\left|x_{0}\right| \leq a_{0}$ there exists $a \in[0, t)$ with

$$
|u(s)|>a_{0} \text { for } s \in(a, t] \text { and }|u(a)|=a_{0} .
$$

Also

$$
|u(s)|^{\prime} \leq\left|u^{\prime}(s)\right| \leq g(s,|u(s)|) \text { a.e. on }(a, t)
$$

So

$$
\left\{\begin{array}{l}
|u(s)|^{\prime} \leq g(s,|u(s)|) \text { a.e. on }(a, t) \\
|u(a)|=a_{0} .
\end{array}\right.
$$

Now a standard comparison theorem for ordinary differential equations in the real case [12, Theorem 1.10.2] guarantees that $|u(s)| \leq r(s)$ for $s \in$ $[a, t]$. In particular $|u(t)| \leq r(t)$. As a result $|u|_{0}<M_{0}$. Next suppose $y$ is a possible solution of (1.6) and assume $y(0)=x_{0} \in M$. Let $t \in[0, T]$ and assume $|y(t)|>a_{0}$. Then there exists $a \in[0, t)$ with

$$
|y(s)|>a_{0} \text { for } s \in(a, t] \text { and }|y(a)|=a_{0} .
$$

Also since $\tau_{\varepsilon}: \mathbb{R}^{n} \rightarrow[0,1]$ we have

$$
|y(s)|^{\prime} \leq\left|y^{\prime}(s)\right| \leq g(s,|y(s)|) \text { a.e. on }(a, t),
$$

and as above we have $|y(s)| \leq r(s)$ for $s \in[a, t]$. In particular $|y(t)| \leq r(t)$. As a result $|y|_{0}<M_{0}$.

Remark 2.1. One could also obtain an analogue of Theorem 2.1 when the solution set lies in $A_{n}[0, T]$ if we use Remark 1.1 .

Theorem 2.2. Suppose $M \subseteq C_{n}[0, T]$ is nonempty and compact (respectively nonempty compact and connected) and assume (1.2), (1.3), (1.10), (1.11) and (2.1) hold. In addition suppose the following conditions are satisfied:

$$
g(t, x) \text { is nondecreasing in } x \text { for a.e. } t \in[0, T]
$$


and

$$
\left\{\begin{array}{l}
\text { the problem } \\
\left\{\begin{array}{l}
v^{\prime}(t)=\left(\sup _{t \in[0, T]} k(t)\right) g(t, v(t)) \text { for a.e. } t \in[0, T] \\
v(0)=\sup \left\{|h|_{0}: h \in M\right\} \\
\text { has a maximal solution } r(t) \text { on }[0, T] .
\end{array}\right.
\end{array}\right.
$$

Then $S_{I}\left(M ; \mathbb{R}^{n}\right)$ is a nonempty compact (respectively nonempty compact and connected) subset of $C_{n}[0, T]$.

Proof. We will apply Theorem 1.2 with $\varepsilon>0$ and $M_{0}=\sup _{t \in[0, T]} r(t)+1$. Let $u$ be a possible solution of (1.9) for any $h \in M$. Then

$$
|u(t)| \leq \sup \left\{|w|_{0}: w \in M\right\}+\left(\sup _{t \in[0, T]} k(t)\right) \int_{0}^{t} g(s,|u(s)|) d s \equiv v(t)
$$

for $t \in[0, T]$. Now (2.3) implies

so

$$
v^{\prime}(t)=\left(\sup _{t \in[0, T]} k(t)\right) g(t,|u(t)|) \leq\left(\sup _{t \in[0, T]} k(t)\right) g(t, v(t)) \text { a.e., }
$$

$$
\left\{\begin{array}{l}
v^{\prime}(t) \leq\left(\sup _{t \in[0, T]} k(t)\right) g(t, v(t)) \text { for a.e. } t \in[0, T] \\
v(0)=\sup \left\{|w|_{0}: w \in M\right\} .
\end{array}\right.
$$

Now [12, Theorem 1.10.2] guarantees that $v(t) \leq r(t)$ for $t \in[0, T]$, so $|u(t)|<M_{0}$ for $t \in[0, T]$. A similar argument guarantees that $|y(t)|<M_{0}$, $t \in[0, T]$, for any possible solution $y$ of (1.13) for any $h \in M$.

\section{Fuzzy differential and integral equations}

We first consider (1.15) where we interpret (1.15) as a family of inclusions (1.16). We denote the solution set of the $\beta^{\text {th }}$ inclusion by $S_{\beta}\left(V_{\beta}\right)$ (the system (1.16) can only have any significance as a replacement for (1.15) if the solutions generate fuzzy sets i.e. if the sets $S_{\beta}\left(V_{\beta}\right)$ are level sets of a fuzzy set $S(V)$ say). Before we state our main result recall a mapping $H:[0, T] \rightarrow E^{n}$ is strongly measurable if each level set mapping $[H(\cdot)]^{\beta}$ : $[0, T] \rightarrow C K\left(\mathbb{R}^{n}\right)$ is measurable.

Theorem 3.1. Suppose $F:[0, T] \times \mathbb{R}^{n} \rightarrow E^{n}$ and assume the following conditions hold:

$$
\begin{gathered}
t \mapsto F(t, x) \quad \text { is strongly measurable for every } x \in \mathbb{R}^{n} \\
x \mapsto F(t, x) \text { is upper semicontinuous for a.e. } t \in[0, T]
\end{gathered}
$$




$$
\begin{aligned}
& V \in C^{n}[0, T] \\
& \left\{\begin{array}{l}
\text { for each } t \in[0, T], k(t, s) \text { is measurable on }[0, t] \text { and } \\
k(t)=\operatorname{ess} \sup |k(t, s)|, 0 \leq s \leq t, \text { is bounded on }[0, T]
\end{array}\right. \\
& \left\{\begin{array}{l}
\text { the map } t \mapsto k_{t} \text { is continuous from }[0, T] \text { to } \\
L^{\infty}\left([0, T], L_{n \times n}^{1}[0, T]\right) ; \text { here } k_{t}(s)=k(t, s)
\end{array}\right. \\
& \left\{\begin{array}{l}
\text { there exists a } L^{1} \text {-Carathéodory function } \\
g:[0, T] \times[0, \infty) \rightarrow[0, \infty) \text { such that } D(F(t, u), \hat{0}) \leq g(t,|u|) \\
\text { for a.e. } t \in[0, T] \text { and all } u \in \mathbb{R}^{n}
\end{array}\right. \\
& g(t, x) \text { is nondecreasing in } x \text { for a.e. } t \in[0, T] \\
& \left\{\begin{array}{l}
\text { for eaxh } \beta \in[0,1], \text { the problem } \\
\left\{\begin{array}{l}
v^{\prime}(t)=\left(\sup _{t \in[0, T]} k(t)\right) g(t, v(t)) \text { for a.e. } t \in[0, T] \\
v(0)=\sup \left\{|h|_{0}: h \in V_{\beta}\right\} \\
\text { has a maximal solution } r_{\beta}(t) \text { on }[0, T]
\end{array}\right.
\end{array}\right. \\
& \left\{\begin{array}{l}
H(t, \cdot, \cdot): \mathbb{R}^{n} \times[0,1] \rightarrow C K\left(\mathbb{R}^{n}\right) \text { given by }(x, \beta) \rightarrow[F(t, x)]^{\beta} \\
\text { is upper semicontinuous for a.e. } t \in[0, T] .
\end{array}\right.
\end{aligned}
$$

and

Then the solution set $S_{\beta}\left(V_{\beta}\right)$ of the family of inclusions (1.16) are the level sets of a fuzzy set $S(V) \in C^{n}[0, T]$.

Remark 3.1. In Theorem 3.1, (3.3) could be replaced by $V \in C_{\star}^{n}[0, T]$ (here $C_{\star}^{n}[0, T]$ denotes the space of normal upper semicontinuous fuzzy sets with compact support over $\left.C_{n}[0, T]\right)$.

Remark 3.2. Of course (3.9) implies (3.2). Note also that (i) $[u]^{\alpha} \subseteq[u]^{\beta}$ for $0 \leq \beta \leq \alpha \leq 1$ and (ii) if $\left(\alpha_{k}\right)$ is a nondecreasing sequence converging to $\alpha>0$ then $[u]^{\alpha}=\bigcap_{k>1}[u]^{\alpha_{k}}$, guarantee that $R(t, x, \cdot):[0,1] \rightarrow C K\left(\mathbb{R}^{n}\right)$ given by $\beta \mapsto[F(t, x)]^{\bar{\beta}}$ is upper semicontinuous for each $(t, x) \in[0,1] \times \mathbb{R}^{n}$. Notice assumption (3.9) is only needed to establish (3.17), so in Theorem 3.1 we could replace (3.9) with the following closure property:

$$
\left\{\begin{array}{l}
\text { for any sequences }\left\{\beta_{i}\right\} \subseteq[0,1],\left\{x_{\beta_{i}}\right\} \subseteq C_{n}[0, T] \\
\left\{u_{\beta_{i}}\right\} \subseteq L_{n}^{1}[0, T] \text { with } \beta_{i} \rightarrow \beta, x_{\beta_{i}} \rightarrow x_{\beta} \text { in } C_{n}[0, T] \\
u_{\beta_{i}} \rightarrow u_{\beta} \text { weakly in } L_{n}^{1}[0, T] \text { with } u_{\beta_{i}}(\cdot) \in F_{\beta_{i}}\left(\cdot, x_{\beta_{i}}(\cdot)\right) \\
\text { a.e., we have } u_{\beta}(t) \in F_{\beta}\left(t, x_{\beta}(t)\right) \text { for a.e. } t \in[0, T]
\end{array}\right.
$$


Proof. We will apply Theorem 1.4 (with Remark 1.2). Let $Y_{\beta}=S_{\beta}\left(V_{\beta}\right)$. Fix $\beta \in[0,1]$. First we claim

$$
Y_{\beta} \text { is nonempty compact and connected. }
$$

To see this we apply Theorem 2.2. Note (with $F$ replaced by $F_{\beta}$ ) (1.2) follows from (3.2) and Theorem 1.5, (1.3) follows from (3.1), (2.1) (see (3.6)) and (2.4) (see (3.8)). Now Theorem 2.2 guarantees that (3.11) is true, so condition (i) of Theorem 1.4 (with Remark 1.2) is satisfied. Let $0 \leq \alpha \leq \beta \leq 1$. Now $V_{\beta} \subseteq V_{\alpha}$ and $F_{\beta} \subseteq F_{\alpha}$ so

$$
Y_{\beta}=S_{\beta}\left(V_{\beta}\right) \subseteq S_{\alpha}\left(V_{\alpha}\right)=Y_{\alpha},
$$

and condition (ii) of Theorem 1.4 (with Remark 1.2) is satisfied. It remains to show condition (iii) of Theorem 1.4. Let $\beta_{i}$ be a nondecreasing sequence in $[0,1]$ with $\beta_{i} \rightarrow \beta$. (As a matter of interest note since $S_{\beta_{i}}\left(V_{\beta_{i}}\right)$ is a nonincreasing sequence of nonempty compact and connected sets then Theorem 1.7 guarantees that $S=\bigcap_{i} S_{\beta_{i}}\left(V_{\beta_{i}}\right)$ is nonempty compact and connected.) We claim

$$
S_{\beta}\left(V_{\beta}\right)=S .
$$

If (3.13) is true then our result follows from Theorem 1.4 (with Remark 1.2). It remains to show (3.13). Now since $S_{\beta}\left(V_{\beta}\right) \subseteq S_{\beta_{i}}\left(V_{\beta_{i}}\right)$ for each $i$ we have $S_{\beta}\left(V_{\beta}\right) \subseteq S$. Now for each $i$ let $x_{\beta_{i}} \in S_{\beta_{i}}\left(V_{\beta_{i}}\right)$. Then there exists a continuous function $v_{\beta_{i}} \in V_{\beta_{i}}$ and an integrable function $u_{\beta_{i}}(\cdot) \in$ $F_{\beta_{i}}\left(\cdot, x_{\beta_{i}}(\cdot)\right)$ with

$$
x_{\beta_{i}}(t)=v_{\beta_{i}}(t)+\int_{0}^{t} k(t, s) u_{\beta_{i}}(s) d s .
$$

Now $\left\{v_{\beta_{i}}\right\}$ is uniformly bounded and equicontinuous from the Arzela-Ascoli theorem (recall $V_{0}$ is compact and $V_{\beta_{i}} \subseteq V_{0}$ ). Now for $t \in[0, T]$ we have

$$
\left|x_{\beta_{i}}(t)\right| \leq \sup \left\{|h|_{0}: h \in V_{0}\right\}+\left(\sup _{t \in[0, T]} k(t)\right) \int_{0}^{t} g\left(s,\left|x_{\beta_{i}}(s)\right|\right) d s \equiv w_{\beta_{i}}(t) .
$$

Then

$$
w_{\beta_{i}}^{\prime}(t)=\left(\sup _{t \in[0, T]} k(t)\right) g\left(t,\left|x_{\beta_{i}}(t)\right|\right) \leq\left(\sup _{t \in[0, T]} k(t)\right) g\left(t, w_{\beta_{i}}(t)\right)
$$

a.e. so

$$
\left\{\begin{array}{l}
w_{\beta_{i}}^{\prime}(t) \leq\left(\sup _{t \in[0, T]} k(t)\right) g\left(t, w_{\beta_{i}}(t)\right) \text { for a.e. } t \in[0, T] \\
w_{\beta_{i}}(0)=\sup \left\{|h|_{0}: h \in V_{0}\right\}
\end{array}\right.
$$


Now [12, Theorem 1.10.2] guarantees that $w_{\beta_{i}}(t) \leq r(t)$ for $t \in[0, T]$; here $r=r_{0}\left(r_{0}\right.$ is as in (3.8)). Thus

$$
\left|x_{\beta_{i}}(t)\right| \leq r(t) \leq \sup _{t \in[0, T]} r(t)=r(T) \text { for } t \in[0, T] .
$$

As a result $\left\{x_{\beta_{i}}\right\}$ is uniformly bounded. Also there exists $h_{r(T)} \in L^{1}[0, T]$ with

$$
|g(t, x)| \leq h_{r(T)}(t) \text { for a.e. } t \in[0, T] \text { and }|x| \leq r(T) .
$$

Now for $t, \tau \in[0, T]$ with $t>\tau$ we have

$$
\begin{aligned}
\left|x_{\beta_{i}}(t)-x_{\beta_{i}}(\tau)\right| \leq & \left|v_{\beta_{i}}(t)-v_{\beta_{i}}(\tau)\right|+\int_{0}^{t}\left|[k(t, s)-k(\tau, s)] u_{\beta_{i}}(s)\right| d s \\
& +\int_{\tau}^{t}\left|k(\tau, s) u_{\beta_{i}}(s)\right| d s \\
\leq & \left|v_{\beta_{i}}(t)-v_{\beta_{i}}(\tau)\right| \\
& +|k(t, \cdot)-k(\tau, \cdot)| L^{\infty} \int_{0}^{t}\left|u_{\beta_{i}}(s)\right| d s \\
& +\left(\sup _{t \in[0, T]} k(t)\right) \int_{\tau}^{t}\left|u_{\beta_{i}}(s)\right| d s \\
\leq & v_{\beta_{i}}(t)-v_{\beta_{i}}(\tau) \mid \\
& +|k(t, \cdot)-k(\tau, \cdot)|_{L^{\infty}} \int_{0}^{t} h_{r(T)}(s) d s \\
& +\left(\sup _{t \in[0, T]} k(t)\right) \int_{\tau}^{t} h_{r(T)}(s) d s .
\end{aligned}
$$

This together with (3.5) and the fact that $\left\{v_{\beta_{i}}\right\}$ is equicontinuous implies that $\left\{x_{\beta_{i}}\right\}$ is equicontinuous. As a result there exists $\left\{x_{\beta_{i(1)}}\right\} \subseteq\left\{x_{\beta_{i}}\right\}$ with $x_{\beta_{i(1)}} \rightarrow x_{\beta} \in C_{n}[0, T]$. In addition there exists $\left\{v_{\beta_{i(2)}}\right\} \subseteq\left\{v_{\beta_{i(1)}}\right\}$ with $v_{\beta_{i(2)}} \rightarrow v_{\beta} \in V_{\beta}$ (clearly), and also of course $x_{\beta_{i(2)}} \rightarrow x_{\beta}$. From (3.15) and (3.16) we have

$$
\left|u_{\beta_{i(2)}}(t)\right| \leq h_{r(T)}(t) \text { a.e., }
$$

so the sequence of functions

$$
w_{\beta_{i(2)}}(t)=\frac{u_{\beta_{i(2)}}(t)}{h_{r(T)}(t)}
$$

belongs to the unit ball of $L_{n}^{\infty}[0, T]$. Note Theorem 1.6 guarantees that the unit ball of $L_{n}^{\infty}[0, T]$ is weak ${ }^{\star}$ compact. Thus there is a subsequence $\left\{w_{\beta_{i(3)}}\right\} \subseteq\left\{w_{\beta_{i(2)}}\right\}$ which converges weak ${ }^{\star}$ to a $w_{\beta} \in L_{n}^{\infty}[0, T]$. Now $\left[4\right.$, p. 14] guarantees that $\left\{u_{\beta_{i(3)}}\right\}$ converges weakly in $L_{n}^{1}[0, T]$ to 
$u_{\beta}=h_{r(T)} w_{\beta}$. Next Theorem 1.3 guarantees that there is a subsequence $\left\{u_{\beta_{i(4)}}\right\} \subseteq\left\{u_{\beta_{i(3)}}\right\}$ with

$$
\int_{0}^{t} k(t, s) u_{\beta_{i(4)}}(s) d s \rightarrow \int_{0}^{t} k(t, s) u_{\beta}(s) d s
$$

This together with (3.14) implies

$$
x_{\beta}(t)=v_{\beta}(t)+\int_{0}^{t} k(t, s) u_{\beta}(s) d s .
$$

We claim

$$
u_{\beta}(t) \in F_{\beta}\left(t, x_{\beta}(t)\right) \text { for a.e. } t \in[0, T] .
$$

If (3.17) is true then (1.16) is satisfied so $x_{\beta} \in S_{\beta}\left(V_{\beta}\right)$. Thus $S \subseteq S_{\beta}\left(V_{\beta}\right)$ and (3.13) is true. It remains to show (3.17). Fix $t \in[0, T]$ and let $\varepsilon>0$ be given. Now (3.9) (assume of course it is holding for this $t$ ) guarantees that there exists a neighborhood $U$ of $\left(x_{\beta}(t), \beta\right)$ such that for $(v, \alpha) \in U$ we have

$$
[F(t, v)]^{\alpha} \subseteq\left[F\left(t, x_{\beta}(t)\right)\right]^{\beta}+\varepsilon B^{\star}
$$

here $B^{\star}$ is the unit ball in $\mathbb{R}^{n}$. Choose $i(4)$ sufficiently large so that $\left(x_{\beta_{i(4)}}(t), \beta_{i(4)}\right) \in U$ and thus

$$
\left[F\left(t, x_{\beta_{i(4)}}(t)\right)\right]^{\beta_{i(4)}} \subseteq\left[F\left(t, x_{\beta}(t)\right)\right]^{\beta}+\varepsilon B^{\star} .
$$

Thus given a neighborhood $N$ of zero there exists $i(5)$ sufficiently large so that $\left(u_{\beta_{i}}(t), x_{\beta_{i}}(t)\right) \in \operatorname{graph}\left(G_{\beta}\right)+N$ for $i \geq i(5)$; here $G_{\beta}(\cdot)=F(t, \cdot, \beta)$ is the map given by $x \mapsto[F(t, x)]^{\beta}$. Now (3.17) holds from the convergence theorem in $[4$, p. 60].

Next we consider (1.17) where we interpret (1.17) as a family of inclusions (1.18). We denote the solution set of the $\beta^{t h}$ inclusion by $S_{\beta}\left(\left[X_{0}\right]^{\beta}, \mathbb{R}^{n}\right)$. Note the system (1.18) can only have any significance as a replacement for (1.17) if the solutions generate fuzzy sets i.e. if the sets $S_{\beta}\left(\left[X_{0}\right]^{\beta}, \mathbb{R}^{n}\right)$ are level sets of a fuzzy set $S\left(X_{0}, \mathbb{R}^{n}\right)$ say.

Theorem 3.2. Suppose $F:[0, T] \times \mathbb{R}^{n} \rightarrow E^{n}$ and assume (3.1), (3.2), (3.6) and (3.9) hold. In addition assume the following conditions are satisfied:

$$
X_{0} \in E^{n}
$$


and

$$
\left\{\begin{array}{l}
\text { for each } \beta \in[0,1], \text { the problem } \\
\left\{\begin{array}{l}
v^{\prime}(t)=g(t, v(t)) \text { for a.e. } t \in[0, T] \\
v(0)=\sup \left\{\left|w_{0}\right|: w_{0} \in\left[X_{0}\right]^{\beta}\right\}
\end{array}\right. \\
\text { has a maximal solution } r_{\beta}(t) \text { on }[0, T] .
\end{array}\right.
$$

Then the solution set $S_{\beta}\left(\left[X_{0}\right]^{\beta}, \mathbb{R}^{n}\right)$ of the family of inclusions (1.18) are the level sets of a fuzzy set $S\left(X_{0}, \mathbb{R}^{n}\right)$ defined on $A_{n}^{1}[0, T]$.

Remark 3.3. It is easy to adjust (3.6) so that $A_{n}^{1}[0, T]$ in the statement of Theorem 3.2 is replaced by $A_{n}[0, T]$.

Remark 3.4. In Theorem 3.2 assumption (3.9) could be replaced by (3.10).

Proof. Let $Y_{\beta}=S_{\beta}\left(\left[X_{0}\right]^{\beta}, \mathbb{R}^{n}\right)$. The proof is essentially the same argument as in Theorem 3.1 with the only significant difference being in checking that if $\beta_{i}$ is a nondecreasing sequence in $[0,1]$ with $\beta_{i} \rightarrow \beta$ then

$$
S=\bigcap_{i} S_{\beta_{i}}\left(\left[X_{0}\right]^{\beta_{i}}, \mathbb{R}^{n}\right) \subseteq S_{\beta}\left(\left[X_{0}\right]^{\beta}, \mathbb{R}^{n}\right) .
$$

To see (3.20) let $x_{\beta_{i}} \in S_{\beta_{i}}\left(\left[X_{0}\right]^{\beta_{i}}, \mathbb{R}^{n}\right)$. There exists $x_{0, \beta_{i}} \in\left[X_{0}\right]^{\beta_{i}}$ and an integrable function $u_{\beta_{i}}(\cdot) \in F_{\beta_{i}}\left(\cdot, x_{\beta_{i}}(\cdot)\right)$ with

$$
x_{\beta_{i}}(t)=x_{0, \beta_{i}}+\int_{0}^{t} u_{\beta_{i}}(s) d s .
$$

Let $t \in[0, T]$ and we will show $\left|x_{\beta_{i}}(t)\right| \leq r(T)$; here $r=r_{0}$. Let $a_{0}=$ $\sup \left\{\left|w_{0}\right|: w_{0} \in\left[X_{0}\right]^{0}\right\}$. If $\left|x_{\beta_{i}}(t)\right| \leq a_{0}$ we are finished so it remains to discuss the case when $\left|x_{\beta_{i}}(t)\right|>a_{0}$. In this case since $\left|x_{\beta_{i}}(0)\right|=\left|x_{0, \beta_{i}}\right| \leq a_{0}$ there exists $a \in[0, t)$ with

$$
\left|x_{\beta_{i}}(s)\right|>a_{0} \text { for } s \in(a, t] \text { and }\left|x_{\beta_{i}}(a)\right|=a_{0} .
$$

Also $\left|x_{\beta_{i}}(s)\right|^{\prime} \leq\left|x_{\beta_{i}}^{\prime}(s)\right|$ a.e. on $(a, t)$ so we have

$$
\left\{\begin{array}{l}
\left|x_{\beta_{i}}(s)\right|^{\prime} \leq g\left(s,\left|x_{\beta_{i}}(s)\right|\right) \text { a.e. on }(a, t) \\
\left|x_{\beta_{i}}(a)\right|=a_{0} .
\end{array}\right.
$$

Now [12, Theorem 1.10.2] guarantees that $\left|x_{\beta_{i}}(s)\right| \leq r(s)$ for $s \in[a, t]$, so $\left|x_{\beta_{i}}(t)\right| \leq r(t)$. Thus

$$
\left|x_{\beta_{i}}(t)\right| \leq r(T) \text { for } t \in[0, T],
$$

so $\left\{x_{\beta_{i}}\right\}$ is uniformly bounded. Also there exists $h_{r(T)} \in L^{1}[0, T]$ with

$$
|g(t, x)| \leq h_{r(T)}(t) \text { for a.e. } t \in[0, T] \text { and }|x| \leq r(T) \text {, }
$$


so for $t, \tau \in[0, T]$ with $t>\tau$ we have

$$
\left|x_{\beta_{i}}(t)-x_{\beta_{i}}(\tau)\right| \leq \int_{\tau}^{t}\left|u_{\beta_{i}}(s)\right| d s \leq \int_{\tau}^{t} h_{r(T)}(s) d s
$$

and as a result $\left\{x_{\beta_{i}}\right\}$ is equicontinuous. Now follow the argument in the proof of Theorem 3.1.

\section{References}

[1] Agarwal, R. P., O'Regan, D., A note on the structure of the solution set for the Cauchy differential inclusion in Banach spaces, in "Set Valued Mappings with Applications in Nonlinear Analysis", (eds. R. P. Agarwal and D. O'Regan), Taylor and Francis Publishers, London, 2002, 11-15.

[2] Agarwal, R. P., O'Regan, D., Lakshmikantham, V., Fuzzy Volterra integral equations: a stacking theorem approach, Appl. Anal. 83 (2004), 521-532.

[3] Agarwal, R. P., O'Regan, D., Lakshmikantham, V., A stacking theorem approach for fuzzy differential equations, Nonlinear Anal. 55 (2003), 299-312.

[4] Aubin, J. P., Cellina, A., Differential Inclusions, Springer Verlag, New York, 1984.

[5] Conway, J., A Course in Functional Analysis, Springer Verlag, New York, 1990.

[6] Corduneanu, C., Integral Equations and Applications, Cambridge University Press, New York, 1991.

[7] Diamond, P., Theory and applications of fuzzy Volterra integral equations, preprint.

[8] Diamond, P., Watson, P., Regularity of solution sets for differential inclusions quasiconcave in parameter, Appl. Math. Lett. 13 (2000), 31-35.

[9] Górniewicz, L., Topological Fixed Point Theory of Multivalued Maps, Kluwer Acad. Publishers, Dordrecht, 1999.

[10] Hüllermeier, E., An approach to modelling and simulation of uncertain dynamical systems, Internat. J. Uncertain. Fuzziness and Knowledge-Based Systems 5 (1997), $117-137$.

[11] Kuratowski, K., Topology, Vol. 2, Academic Press, New York, 1968.

[12] Lakshmikantham, V., Leela, S., Differential and Integral Inequalities, Vol. I, Academic Press, New York, 1969.

[13] Lakshmikantham, V., Mohapatra, R., Theory of Fuzzy Differential Equations and Inclusions, Taylor and Francis Publishers, London, 2003.

[14] Negoita, C. V., Ralescu, D. A., Applications of Fuzzy Sets to System Analysis, John Wiley \& Sons, New York, 1975.

RAVi P. AgarWAL

DONAL O'REgAN

Department of Mathematical Sciences Department of Mathematics Florida Institute of TeChNOLOGY NATIONAL University OF IRELAND

Melbourne, FL 32901-6975

GALWAY

USA

IRELAND

E-MAIL: AGARWAL@FIT.EDU

E-MAIL: DONAL.OREGAN@NUIGALWAY.IE 
V. LAKSHMIKANTHAM

Department of Mathematical Sciences

Florida Institute of TECHNOLOGY

Melbourne, FL 32901-6975

USA

E-MAIL: LAKSHMIK@FIT.EDU 